\title{
Theoretical and experimental study on cavitation bubble dynamics induced by underwater shock wave focusing
}

\author{
Zhenfu Zhang ${ }^{1, a}$, Xiaopeng Kong ${ }^{2, b}$ \\ (1. College of Science, National University of Defense Technology, Changsha 410073, China, \\ 2. Institute of Naval Aeronautical Engineering, Yantai 264001, China) \\ azhangzhenfu198206@163.com, bokosky1987@sina.com
}

Keywords: Shock Wave Focusing; Cavitation Bubbles; High-speed Photography;

\begin{abstract}
Shock wave focusing can produce higher pressure and induce cavitation behaviour in the focal region. Experimental study was carried out based on an underwater shock wave focusing system and an optical arrangement for taking high-speed photographs. The physical process of the cavitation behaviour and the relation between bubble dynamics and pressure were analyzed by comparing between the pressure-time histories and the high-speed photographs. The bubble dynamics was modeled with modified Rayleigh-Plesset equation; the results indicate that the characteristic of bubble dynamics and the duration of bubble behaviour are sensitive to pressure field, and experimental results and theoretical results agree well.
\end{abstract}

\section{Introduction}

Shock wave focusing can produce higher pressure and negative pressure. There is a tensile failure (or cavitation threshold) in water [1]. When the negative is low enough, it may induce cavitation phenomena in the focal region, and cavitation bubbles may appear [2]. Shock wave will be generated when cavitation bubbles collapse. If the stress wave interacts with a solid, it may cause damage to the solid. The interaction between shock wave and bubble contributes to urinary tract stone disintegration and tissue damage in ESWL [3].

The investigation to the shock wave focusing and its induced cavitation is very important for the underwater disintegration and damage. Experimental study was carried out based on an underwater shock wave focusing system and an optical arrangement for taking high-speed photograph, and cavitation phenomena was found in the focal region. The physical process of the cavitation behaviour and the relation between bubble dynamics and pressure were analyzed by comparing between the pressure-time history and the high-speed photographs. The bubble dynamics was modeled with modified Rayleigh-Plesset equation; the results indicate that the characteristic of bubble dynamics and the duration of bubble behaviour are sensitive to pressure field, and the variable tendency of the bubble radius are same in the experiment and theoretical modeling with modified Rayleigh-Plesset equation.

\section{Experimental study}

\subsection{Experimental facility and method}

An underwater shock wave focusing system was set up based on pulsed discharge and ellipsoidal reflector. The configuration of ellipsoidal reflector is shown in Fig.1, the truncated reflector is made of a material with a high acoustical impedance, a semi-major axis $a=500 \mathrm{~mm}$, a semi-minor axis $b=250 \mathrm{~mm}$, and a half-focal length $c=433 \mathrm{~mm}$. The discharge electrodes are mounted at the first focus $F_{1}$ of the ellipsoidal reflector.

Based on the linear acoustic theory and the geometrical characteristic of the ellipsoid, for the acoustic waves generated by the source at the first focus $F_{1}$ of the ellipsoid, the reflection angle $\beta$ of the acoustic ray is equal to the incidence angle $\alpha$ in the inner surface of the reflector, and the acoustic wave rays reflected from the inner surface of the reflector will focus at the second focus $F_{2}$ and high energy density pulse pressure will be produced in the focal region. 

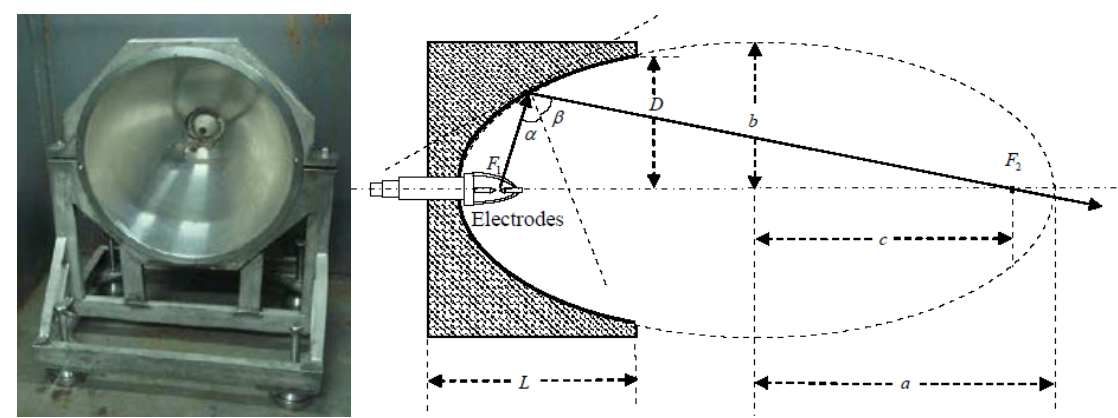

Fig. 1 Configuration of ellipsoidal reflector

\subsection{Experimental results and discussion}

The experimental investigations on shock wave focusing were made in a water basin with $2000 \mathrm{~mm} \times 1000 \mathrm{~mm} \times 1000 \mathrm{~mm}$ filled with tap water. The exporting high voltage was $18 \mathrm{kV}$.

Fig. 2 shows the photographs of bubble cloud near the second focus. It is noticeable that band of cavitation is presented, and no significant bubble motion was observed in the experiments. The variety of pressure during cavitation is shown in Fig.3. Comparing the photograph with the pressure-time history, a tension wave was found, following the compression wave, and a negative pressure formed and cavitation phenomena appeared. At first, the bubbles expanded under the negative pressure, and then decayed when the negative pressure disappeared. The cavitation phenomena persisted about $0.76 \mathrm{~ms}$.

The radius of bubbles can be gotten by dealing with the high-speed photographs of bubble cloud. Because the time of cavitation phenomena persisting is very short, the displacement of the bubbles caused by shock wave and gravity during this period could be ignored and the positions of the bubbles are fixed.

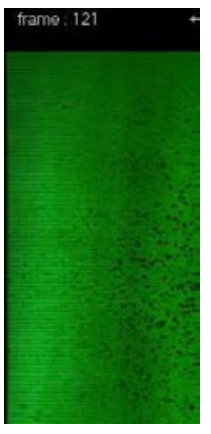

$2.276 \mathrm{~ms}$

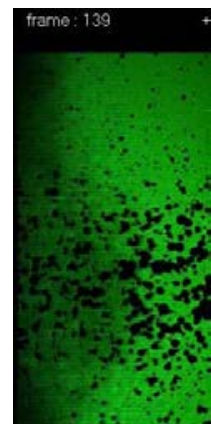

$2.609 \mathrm{~ms}$

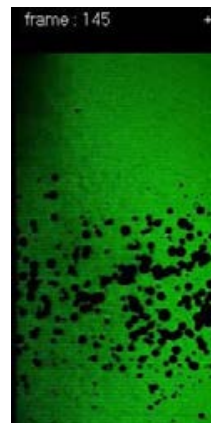

$2.720 \mathrm{~ms}$

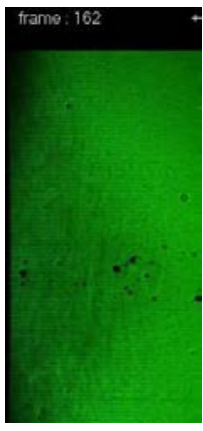

$3.035 \mathrm{~ms}$

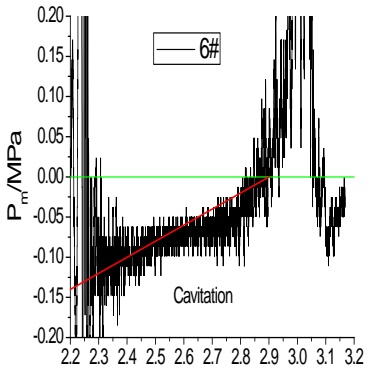

t/ms

Fig. 2 High-speed photographs of bubble cloud in the focal

Fig. 3 Pressure-time history

Fig. 4 shows the collapse time against the maximum radius of bubbles. The bigger the collapse time is, the longer the collapse time is. There is a linear relationship between the maximum bubble radius and the collapse time, but the experimental data are not satisfied with the linear relationship strictly due to the interaction of the bubble cloud and the interaction between the shock wave and the bubbles. Fig. 5 shows time variation of bubble radius, the maximum radiuses of three bubbles are $0.95 \mathrm{~mm}, 0.81 \mathrm{~mm}$ and $0.67 \mathrm{~mm}$. The results indicate that the bubble will expand under tension wave, and reach the maximum radius, and then will decay under compression wave until disappear. The time of bubble growth is longer than the time of bubble decay because the pressure of the surrounding liquid in the growth period is lower than in decay period. 


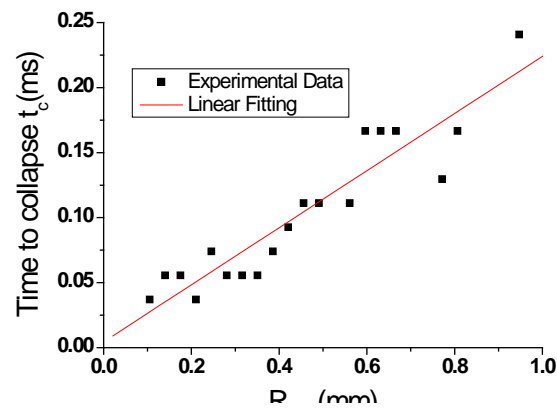

Fig. 4 Collapse time against the maximum radius

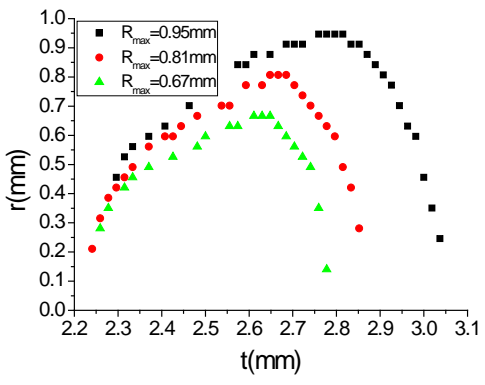

Fig. 5 Time variation of bubble radius

\section{Theoretical modeling}

The modified Rayleigh-Plesset[4,5] can be derived from continuity equation and momentum equation, given as

$$
\begin{aligned}
& R \ddot{R}\left\{1+M+\frac{1}{2} M^{2}+\frac{h(0, \tau)}{c^{2}}+\frac{b}{\rho^{2}} \frac{1}{c^{4}} \frac{\partial p}{\partial \tau}\right\}+\frac{3}{2} \dot{R}^{2}\left\{[1+M]\left[1+\frac{2}{3} M\right]+\frac{R}{2 c^{2}} \frac{\partial c}{\partial \tau}\right\}-h(0, \tau)\left\{[1+M][1+2 M]-\frac{R}{c^{2}} \frac{\partial c}{\partial \tau}\right\} \\
& -\frac{\partial h(0, \tau)}{\partial \tau} \frac{R}{c}[1+M]+\frac{b}{\rho^{2} c^{2}}\left\{\begin{array}{l}
\left.\left[\frac{R}{c^{2}} \frac{\partial c}{\partial \tau}-(1+2 M)(1+M)\right] \frac{\partial p}{\partial \tau}\right\} \\
-\rho R c(1+M) \frac{\partial}{\partial \tau}\left(\frac{1}{\rho c^{2}} \frac{\partial p}{\partial \tau}\right)
\end{array}\right\}=0
\end{aligned}
$$

And the condition on the normal stresses is

$$
p=P_{v}+\left(p_{0}+\frac{2 \sigma}{R_{0}}\right)\left(\frac{R_{0}}{R}\right)^{3 K}-\frac{2 \sigma}{R}-4 \eta \frac{\dot{R}}{R}
$$

The enthalpy $h$ is expressed as

$$
h(x, \tau) \equiv \int_{p(\infty, \tau)}^{p(x, \tau)} \frac{1}{\rho} d p
$$

$\sigma=0.0725 \mathrm{~N} / \mathrm{m}$ is the surface tension, $\eta=0.001 \mathrm{~Pa} \cdot \mathrm{s}$ is the shear viscosity of the liquid, $c$ is sound speed, and $\mathrm{M}$ is mach number.

To close the equations, an equation of state must be used, the modified Tait form

$$
\frac{p+B}{p(\infty)+B}=\left(\frac{\rho}{\rho_{\infty}}\right)^{n}
$$

Where $p(\infty)$ and $\rho_{\infty}$ are undisturbed pressure and liquid density, and the values $B=304.91 \mathrm{MPa}$, $n=7.15$.

The features of the dynamics of bubble can be gotten by solving the equations above using four-order Runge-Kutta method. The Bubble radius as a function of time is shown in Fig.6. We can find that bubble can not rebound several times under pressure field like above, even collapse in a period's time. The results indicate that the characteristic of bubble dynamics and the duration of bubble behaviour are sensitive to pressure field.

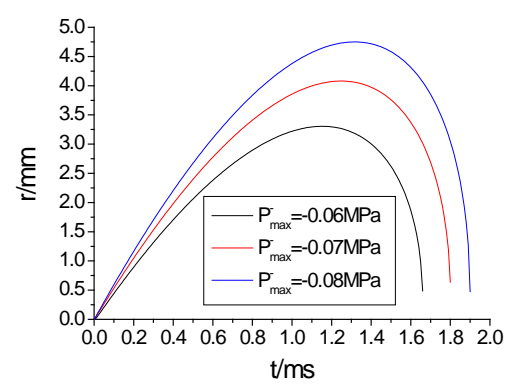

Fig. 7 Time variation of bubble radius under pressure field 


\section{Conclusions}

The higher pressure pulse and the dynamic damage are the important application of underwater shock wave focusing. So the pressure waveforms at the focus and the cavitation phenomena are emphases of the study. The results obtained from the study are summarized as following:

Higher peak pressure can be gotten by the shock wave focusing system, and tension wave and negative pressure can be generated in the focal region. When the negative pressure is lower than the tension threshold of water, cavitation phenomena will appear. The radius of bubble increases first and then decreases; there is a linear relationship between the maximum bubble radius and the collapse time, following Lord Rayleigh's theory; the time of bubble growth is longer than the time of bubble decay, and the characteristic and the duration of bubble dynamics are sensitive to pressure.

\section{Acknowledgement}

This work was supported by grants from the Basic research fund of National University of Defense Technology (JC14-02-07)

\section{References}

[1] S.W.J. Brown, P.R. Williams. The tensile behaviour of elastic liquids under dynamic stressing, J. Non-Newtonian Fluid Mech. 2000, 90: 1-11.

[2] Y. Tomita, T. Obara, et al. Cavitation phenomena in extracorporeal microexplosion lithotripsy, Shock Waves. 1994, 3: 149-157.

[3] I. Chilibon, M. Wevers, et al. Cavitation role in extracorporeal shock wave lithotripsy, Journal of optoelectronics and advanced materials.2006, 8(1): 235-237.

[4] Evert Klaseboer, Boo Cheong Khoo. A modified Rayleigh-Plesset model for a non-spherically symmetric oscillating bubble with applications to boundary integral methods, Engineering Analysis with Boundary Elements. 2006, 30: 59-71.

[5] Lord Rayleigh. On the pressure developed in a liquid during the collapse of a spherical cavity, Philosophical Magazine. 1917, 34(200): 94-98. 